\title{
Analysis of the Light Element Sensitivity and Measurement Time of the Multiple EDX Pole Shoe Detector "Rococo2"
}

\author{
A.Liebel $^{1}$, M. Bornschlegl ${ }^{2}$, R.Eckhardt ${ }^{2}$, S.Jeschke $^{2}$, A.Niculae $^{2}$, H.Soltau $^{1}$ \\ ${ }^{1}$ PNSensor GmbH, Römerstr. 28, D-80803 München, Germany \\ ${ }^{2}$ PNDetector GmbH, Emil-Nolde-Str.10, D-81735 München, Germany
}

Many challenges have to be accomplished for energy-dispersive X-Ray spectroscopy detectors to achieve optimum working conditions in fields like Transmission Electron Microscopy (TEM) or Low Voltage Scanning Electron Microscopy (LVSEM). The attainable X-Ray intensities are weak and the measurement times for collecting a sufficiently high amount of data are long. Detectors with large sensitive area and optimized geometries which cover a high solid angle have to be used to manage these problems. The 4-channel cloverleaf shaped monolithic Silicon Drift Detector named Rococo2 is an ideal candidate for this purpose because it can be positioned right underneath the pole piece and therefore extremely close to the sample. The primary beam is guided through a center hole of $\varnothing 2.4 \mathrm{~mm}$ in the detector chip and the four $15 \mathrm{~mm}^{2}$ large kidney shaped segments of the SDD guarantee a very large solid angle up to $1.4 \mathrm{sr}$. [1]

Comparing this solid angle to the achievable maximum of a conventional $10 \mathrm{~mm}^{2}$ SDD detector which is something in between 0.005 to $0.01 \mathrm{sr}$, it is very obvious that the Rococo 2 detector can deliver a signal more than 100 times higher in intensity at the same measurement time and conditions. This is extremely useful for increasing the count rate when working with thin samples (TEM or STEM) or sensitive SEM samples where the exciting beam current has to be limited. Figure 1 shows EDX mappings of a duplex brass sample. The left image contains about $5 \times 10^{5}$ counts which can be collected with a conventional $10 \mathrm{~mm}^{2}$ SDD with $0.01 \mathrm{sr}$ at a primary beam energy of $20 \mathrm{keV}$ and a beam current of $300 \mathrm{pA}$ in about 10 minutes. The right image contains about $5 \times 10^{7}$ counts and can be taken at the same condition and acquisition time with a Rococo 2 detector with a solid angle $>1.0 \mathrm{sr}$. The benefit in image quality due to the higher contrast is very obvious.

Since the detector is positioned so close to the primary beam, a magnetic electron trap as it is used for conventional SDDs cannot be used because it would disturb the electron beam. Hence, filtering out the high energetic backscattered electrons (BSE) has to be done by means of hardware filters. Foils that adequately stop the BSE up to medium or high energies will also absorb most of the low energy X-Ray photons. However, an appropriate combination of different filter foils for each two detector cells (see figure 2) can still deliver a sufficiently high transmission for the X-Rays over a wide energy range. Figure 3 a) shows transmission curves of a $2 \mu \mathrm{m}$ thick Mylar foil and a $2 \mu \mathrm{m}$ thick Be foil, both capable of stopping electrons with energies up to $12 \mathrm{keV}$. With the resulting transmission of this filter combination a continuous sensitivity down to the carbon and boron line can be achieved. This is further illustrated in figure $3 \mathrm{~b}$ ) which shows spectra from a Mn-Target simultaneously measured with the two different filters and the resulting summarized spectra.

We will present an optimized compact design of the Rococo 2 detection system and measurements with different filter combinations emphasizing the quantum efficiency and the acquisition time benefit. 
[1] A. Niculae et al., Microscopy \& Microanalysis, vol. 18, S2 (2012), pp. 1202-1203
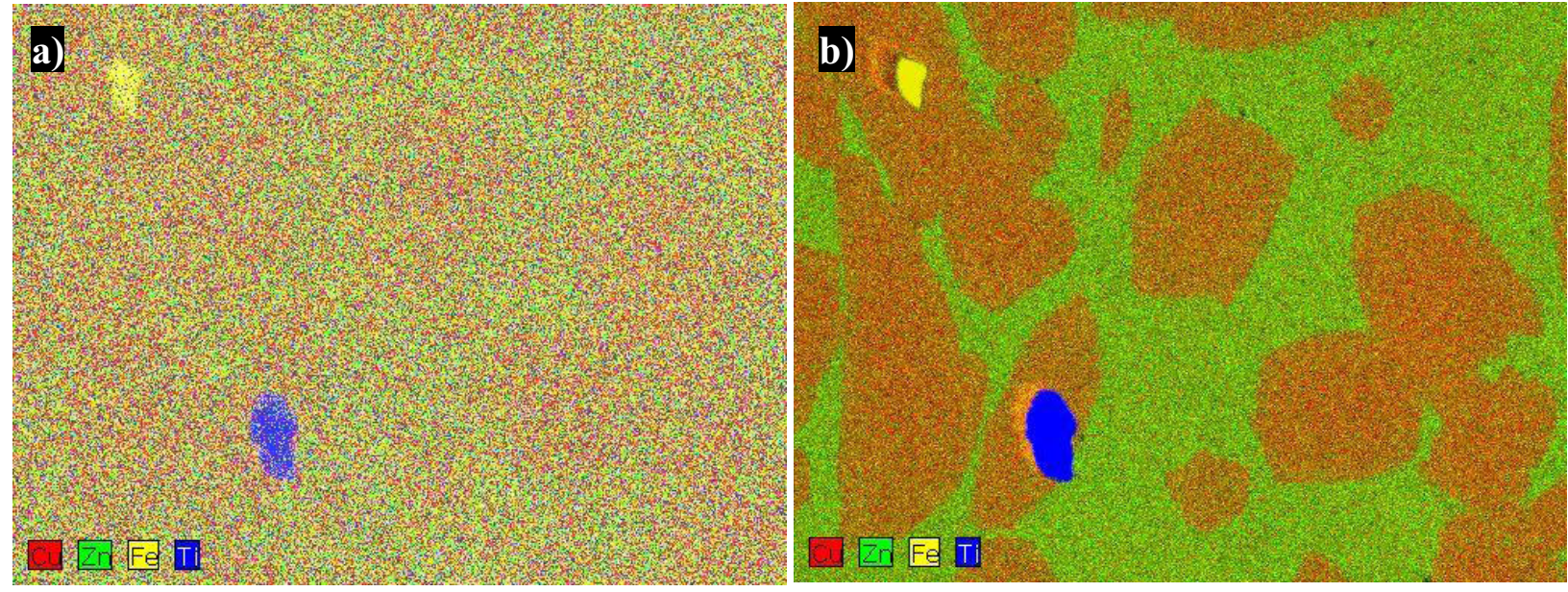

Fig. 1. EDX Mapping of a duplex brass sample showing $\alpha$ and $\beta$ phases with different concentration of copper and zinc. a) Image containing about $5 \times 10^{5}$ counts (conventional $10 \mathrm{~mm}^{2} \mathrm{SDD}$ with $0.01 \mathrm{sr}$ solid angle) and b) Image containing about $5 \times 10^{7}$ counts which can be collected with a Rococo2 Detector at the same acquisition time and conditions. Images are shown without any post-editing.

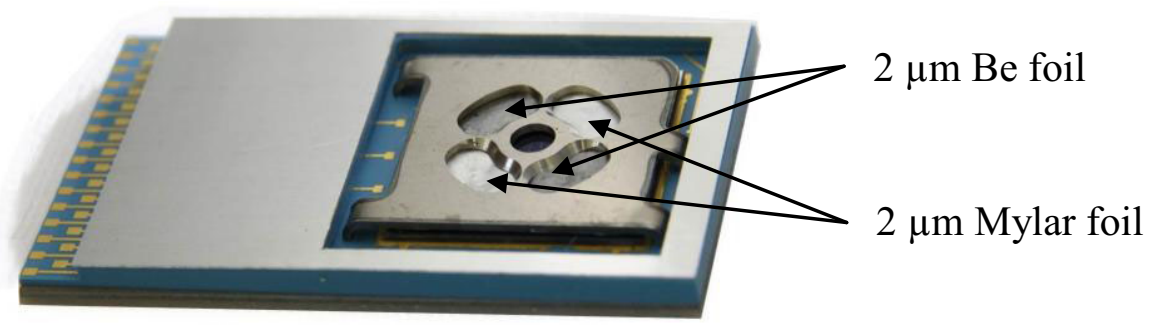

Fig. 2. Rococo2 Detector with a filter combination of $2 \mu \mathrm{m}$ Be and $2 \mu \mathrm{m}$ Mylar
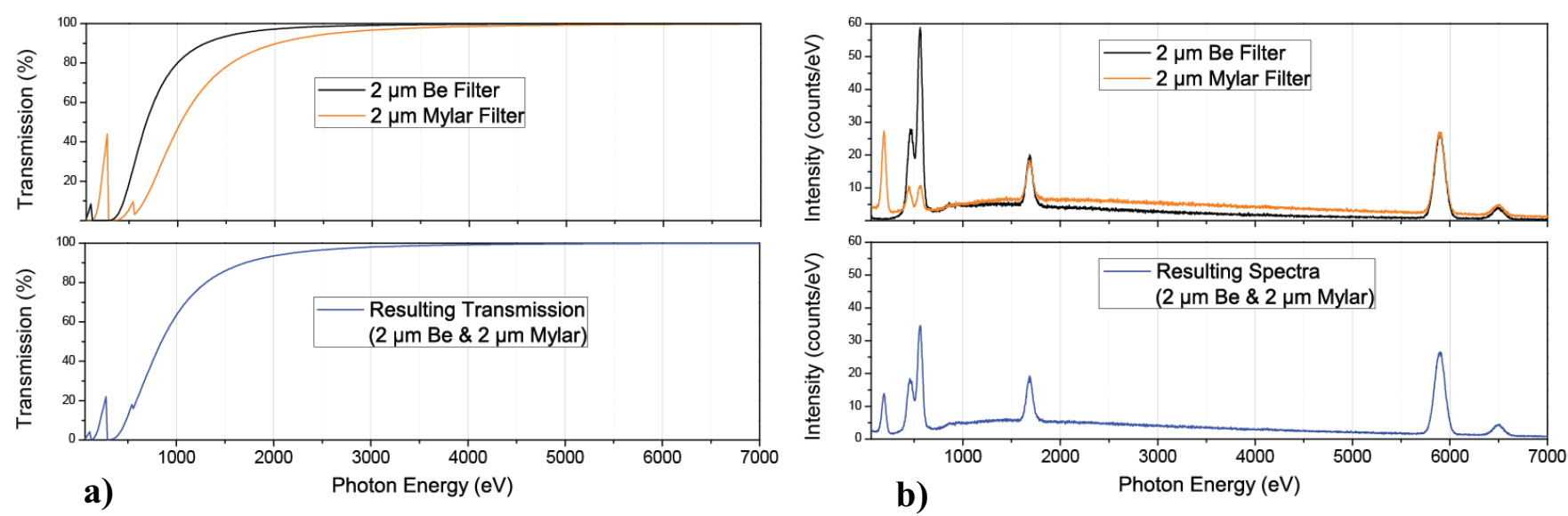

Fig. 3. a) Calculated transmission curves of a $2 \mu \mathrm{m}$ Be and a $2 \mu \mathrm{m}$ Mylar filter and for both filters in combination. b) Measured spectra containing the C-K, O-K, Mn-L, Si-K and Mn-K lines, recorded simultaneously with the two different single filters and the resulting summarized spectra for the combination of both filters (see fig. 2) showing a continuous sensitivity from carbon to manganese. 